\title{
MODEL PREDICTIVE CONTROL OF SHELL BENCHMARK PROCESS
}

\author{
Shiraz Amjad and H. N. Al-Duwaish \\ King Fahd University of Petroleum and Minerals, \\ Department of Electrical Engineering \\ Dhahran, Saudi Arabia \\ \{shiraz, hduwaish\}@kfupm.edu.sa
}

\begin{abstract}
Simultaneous control of overhead and bottoms composition in a binary distillation column using reflux and steam flow as the manipulated variables often proves to be particularly difficult because of the interaction inherent in the process. The development of model based control (MPC) theory in the past 20-25 years has provided a framework which caters for the above distillation control problem. MPC technology has penetrated a significant portion of the process industries and harvested large economical benefits. In this paper model predictive controller is proposed for the control of a two-input-two-output high purity shell distillation column. The use of this controller allows for the impurity and pressure control to be decoupled while simultaneously satisfying column operating conditions. For this purpose the dynamic process model is identified using classical least squares technique.
\end{abstract}

\section{INTRODUCTION}

Model Predictive Control (MPC) has developed considerably over the last few years, both within the research control community and in industry $[1,2,3]$. It integrates optimal control, stochastic control, control of processes with dead time and multivariable control. The term Model Predictive Control does not designate a specific control strategy but a very ample range of control methods, which make an explicit use of a model of the process to obtain the control signal by minimizing an objective function. These predictive controllers are based on a prediction of the future behavior of the process, forecasted using this model of the process. This makes process identification an important sub-problem in the design of model predictive controller.

In this contribution, MPC technique is utilized for the control of a high purity distillation column described by Cott [4]. The control objectives are to maintain the impurity level in the bottoms stream at a certain value while simultaneously preventing over-pressuring of the column.

This paper is structured as follows. In section 2 the considered high purity distillation column is briefly presented and the results of plant model identification are shown. Section 3 contains a short outline of the control objectives for the column and the performance of the designed controller is discussed.

\section{IDENTIFICATION}

In this paper the control of a high purity binary distillation column with 35 trays (see Figure 1) is considered. The main purpose of the binary column is to remove a lightkey impurity from the column feed stream such that the impurity does not reach a downstream reaction system [5].

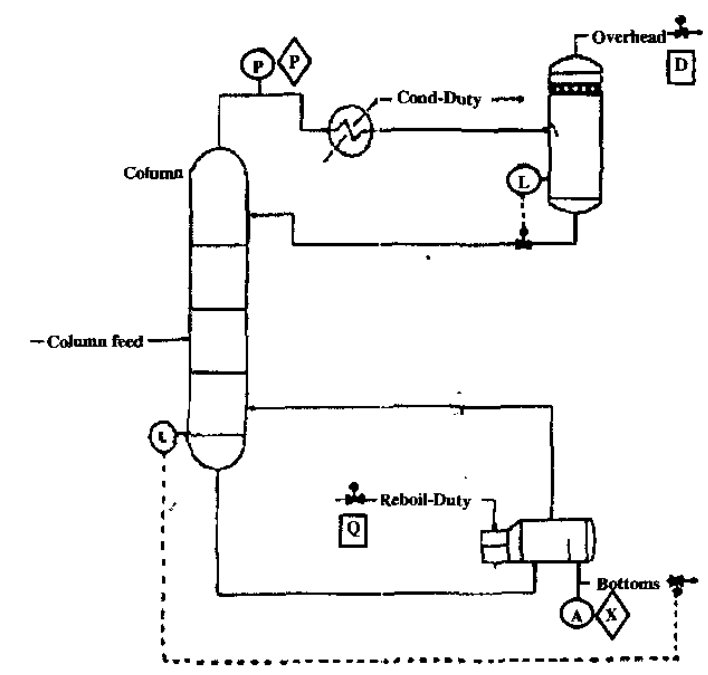

Figure 1 Shell High Purity Distillation Column

Table-1 surnmarizes the typical operating conditions for the column where the inputs (manipulated variables) are overhead flow $(D)$ and reboiler duty $(Q)$ and the outputs (controlled variables) are column pressure $(P)$ and product impurity $(X)$. In this work it is assumed that the feed rate is always constant. It is required that under normal operating conditions all variables should be working at their nominal set points. This is discussed further in section 3 . 
Table 1 Summary of typical benchmark operating conditions

\begin{tabular}{|c|c|c|}
\hline Variable & $\begin{array}{c}\text { Nominal Set } \\
\text { points }\end{array}$ & Normal Operation \\
\hline Pressure $\mathrm{P}$ & 2800 & $2700 \leq \mathrm{P} \leq 2900$ \\
\hline Composition X & 500 & $250 \leq \mathrm{X} \leq 1000$ \\
\hline Distillate D & 20 & $10 \leq \mathrm{D} \leq 30$ \\
\hline Reboiler Q & 2500 & $2000 \leq \mathrm{Q} \leq 3000$ \\
\hline
\end{tabular}

The process models in equations 1 and 2 are obtained from plant tests and are described by Cott [5].

$$
\begin{aligned}
P^{d}(k)= & \frac{-0.6096+0.4022 q^{-1}}{1-1.5298 q^{-1}+0.5740 q^{-2}} D^{d}(k) \\
& +\frac{0.14397-0.0918 q^{-1}}{1-1.5298 q^{-1}+0.5740 q^{-2}} Q^{d}(k) \\
& +\frac{N_{s}}{1-1.5945 q^{-1}+0.5945 q^{-2}} e_{P}(k),
\end{aligned}
$$

where $P^{d}, D^{d}$, and $Q^{d}$ are deviation variables for pressure, overhead flow and reboiler duty respectively, and $e_{p}(k)$ is the white noise input with standard deviation 1.231. The parameter $\mathrm{N}_{\mathrm{s}}$ is used to set the noise level.

The model of the impurity response $(X)$ is slightly nonlinear. The standard deviation of the white noise input $e_{x}(t)$ is 0.667 . It is noted here that the overhead impurity (D) has no affect on distillate flow.

$$
\begin{aligned}
X(k)= & 0.0765 \frac{500000}{Q(k-7)-1500}+0.9325 X(k-1) \\
& +\frac{N_{s}}{1-1.6595 q^{-1}+0.6595} e_{x}(k) .
\end{aligned}
$$

Based on the above information the following discrete time process transfer function model is assumed.

$$
\left[\begin{array}{c}
P \\
X
\end{array}\right]=\left[\begin{array}{ll}
\frac{B_{11}\left(q^{-1}\right)}{A_{11}\left(q^{-1}\right)} & \frac{B_{12}\left(q^{-1}\right)}{A_{12}\left(q^{-1}\right)} \\
\frac{B_{21}\left(q^{-1}\right)}{A_{21}\left(q^{-1}\right)} & \frac{B_{22}\left(q^{-1}\right)}{A_{22}\left(q^{-1}\right)}
\end{array}\right]\left[\begin{array}{l}
D \\
Q
\end{array}\right]+\left[\begin{array}{l}
d_{1} \\
d_{2}
\end{array}\right] .
$$

The polynomials $A_{i j}$ and $B_{i j}$ are of arbitrary order with a form $A=\left(1-a_{1} q^{-1}-a_{2} q^{-2}-\ldots\right)$ and $B=q^{-n}\left(b_{1}+b_{2} q^{-1}+\ldots\right)$, where the integer $n$ is equal to $(d+1)$ where $d$ is the process time delay.

Although models for the process transfer functions and models for the disturbance sequences could be identified simultaneously, we have elected to use the disturbance models as provided by Shell [4]. For singleinput single-output (SISO) identification approach 3500 data points are collected with random step excitation assuming that the plant is already in steady state. For off line simulation, this means that the outputs are allowed to reach steady state before applying the inputs keeping in mind the normal operating constraints of Table 1 . The outputs are shown in figures 2 and 3.

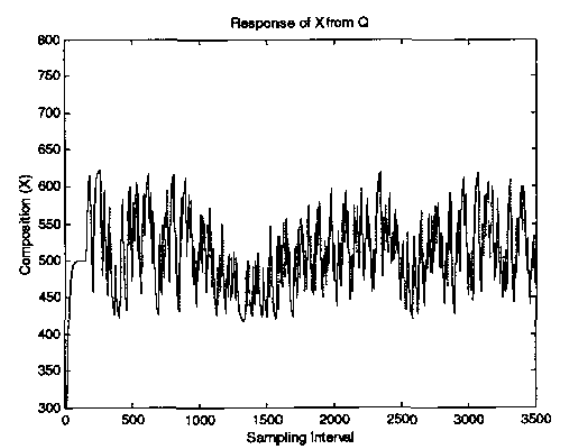

Figure 2 Column Bottom Purity $X$

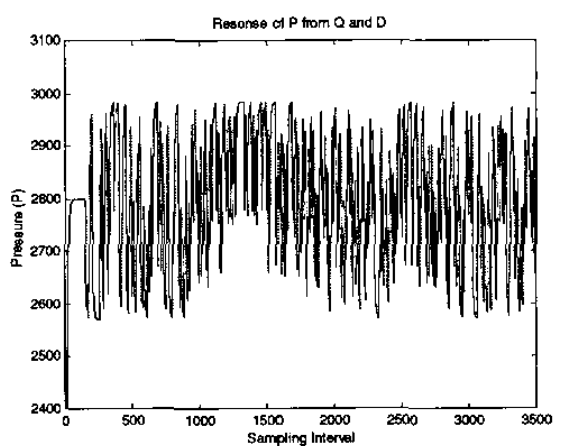

Figure 3 Column Pressure $P$

Estimation of the parameters in the first-order SISO transfer functions in equation 3 is done using classical least squares technique. The identified process model is given by equation 4 . Model validation is performed by comparing the step responses with the actual process models of equations 1 and 2. The results are presented in figures 4,5 and 6 which show that the identified model closely approximates the real plant.

$$
\begin{aligned}
{\left[\begin{array}{l}
P \\
X
\end{array}\right]=} & {\left[\begin{array}{cc}
\frac{-0.5994}{1-0.8722 q^{-1}} & \frac{0.1288}{1-0.8888 q^{-1}} \\
0 & \frac{-0.03965 q^{-7}}{1-0.925 q^{-1}}
\end{array}\right]\left[\begin{array}{l}
D \\
Q
\end{array}\right] } \\
& +\left[\begin{array}{c}
\frac{N_{s}}{1-1.5945 q^{-1}+0.5945 q^{-2}} \\
\frac{N_{s}}{1-1.6595 q^{-1}+0.6595}
\end{array}\right]\left[\begin{array}{l}
e_{P}(k) \\
e_{x}(k)
\end{array}\right]
\end{aligned}
$$

It is worth pointing out here that the identified models provide some useful information regarding the process itself i.e. an increase in the overhead vapor flow $(D)$ shall result in a decrease in the overhead pressure $(P)$, and may 
result in a decrease in the bottoms impurity $(X)$. Similarly an increase in the reboiler duty $(Q)$ shall result in an increase in $P$ and a decrease in $X$.

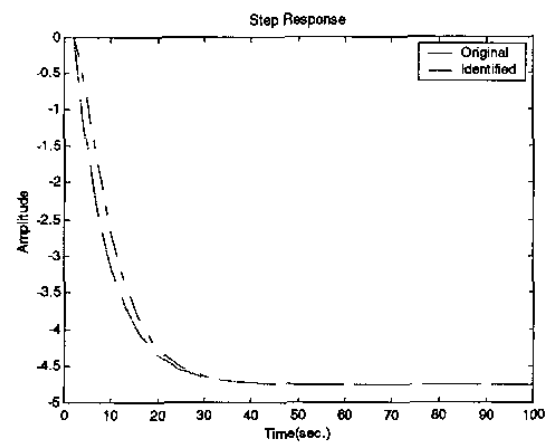

Figure 4 Unit step response of $P$ from $D$

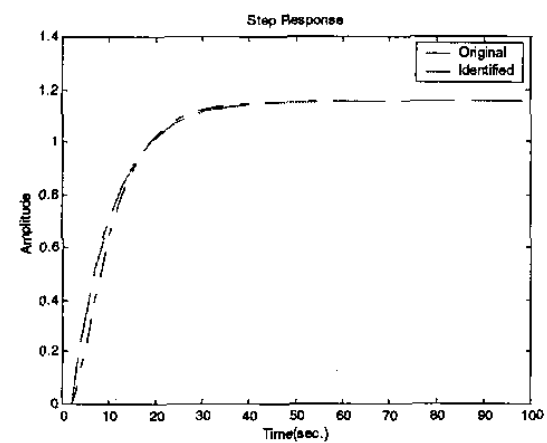

Figure 5 Unit step response of $P$ from $Q$

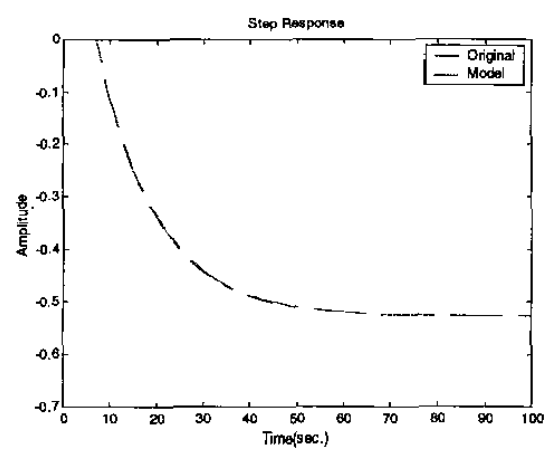

Figure 6 Unit step response of $X$ from $Q$

\section{MODEL PREDICTIVE CONTROL}

For many reasons, distillation remains the most important separation technique in chemical process industries around the world $[6,7,8]$. Improved distillation control can have a significant impact on improving product quality, energy consumption and protecting environmental resources. Our intention in this paper is to demonstrate that MPC technique can be used successfully for the control of large scale processes such as distillation columns working under process constraints and disturbances. Before proceeding to the design of predictive controller for the shell benchmark (distillation column) process understudy a short review of MPC is given as under.

Model Predictive Control (MPC) is probably the most important approach to the advanced control of complex interacting industrial processes. MPC scheme can be viewed as a prediction algorithm or a controller that predicts the behavior of the process outputs over some output horizon $H_{P}$ when the manipulated inputs are changed over some input horizon $H_{c}$. The task of the controller is to compute the present and future manipulated variable moves (inputs) such that the predicted outputs follow the set points in a desirable manner. The controller takes into account the constraints on the inputs and outputs as are given in Table 1. The cost function for this particular process is defined as in equation 5 , which penalizes the deviations of the predicted controlled outputs $\hat{P}$ and $\hat{X}$ denoted by $\hat{z}$ from the nominal set points denoted by $r$ respectively (refer to Table 1). Here $k$ is time of measurements and $Q$ and $R$ are the weighting matrices on the predicted output and change in the controls (inputs).

$$
J(k)=\sum_{i=1}^{H_{\mathrm{p}}}\|\hat{z}(k+i)-r(k+i)\|_{Q(i)}^{2}+\sum_{i=0}^{H_{t^{c}-1}}\|\Delta \hat{u}(k+i)\|_{R(i)}^{2} .
$$

For the shell high purity distillation column the control objectives summarized in Table 1 may be stated simply as follows [5].

- The impurity level $(X)$ in the bottoms stream must be maintained below a certain value (500 units) as set by the ability of the downstream reaction process to tolerate the impurity.

- Because the heat removal is constrained, the column pressure $(P)$ will float with the incoming cooling water temperature and flow. It is therefore important that the controller maintains the column pressure in order to prevent over pressuring of the column and activation of the safety systems.

- Because the overhead material is recycled back upstream, it is required that the flow of this stream $(D)$ is minimized.

With the above objectives predictive controller with a prediction horizon $\mathrm{H}_{\mathrm{p}}=40$, control horizon $\mathrm{H}_{\mathrm{c}}=5$ and a sampling interval of 2 seconds is designed. The diagonal weighting matrix $Q$ and $R$ were chosen as

$$
Q=\left[\begin{array}{ll}
1 & 0 \\
0 & 2
\end{array}\right] \text { and } R=\left[\begin{array}{ll}
2 & 0 \\
0 & 2
\end{array}\right]
$$

Figures 9 and 10 show that the controller provides a desirable performance with the control inputs kept well within the constraints of Table 1 . Figures 7 and 8 
demonstrate stable and quick tracking response of the predictive controller. The process outputs reach their steady state in less than 10 seconds. There is some small overshoot in output $P$ but nevertheless the controller is effective in realizing automatic and optimal benchmark column operation.

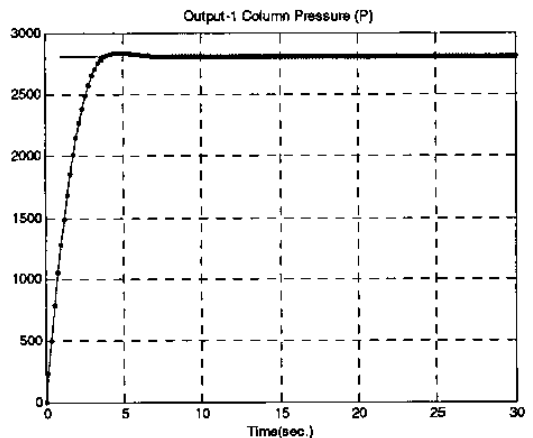

Figure 7 Model predictive control of the column pressure $P$

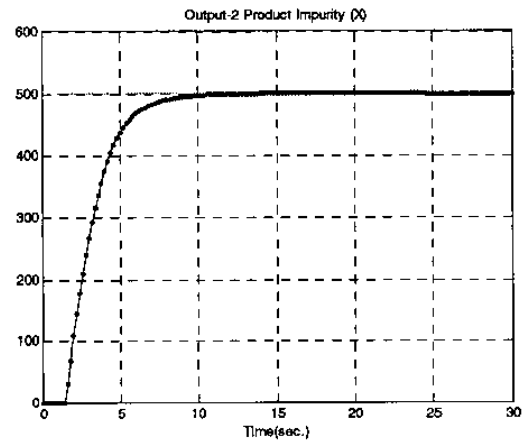

Figure 8 Model predictive control of the impurity level $X$

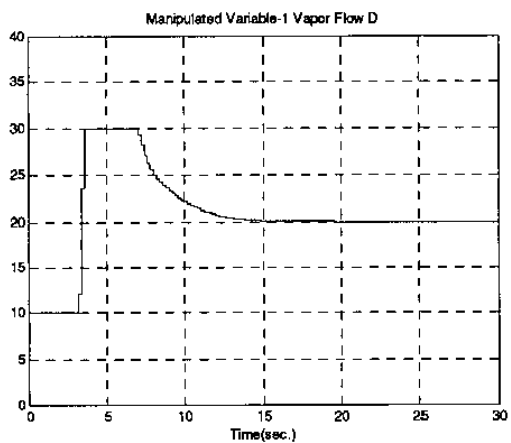

Figure 9 Manipulated Variable Moves for Vapor Flow $D$

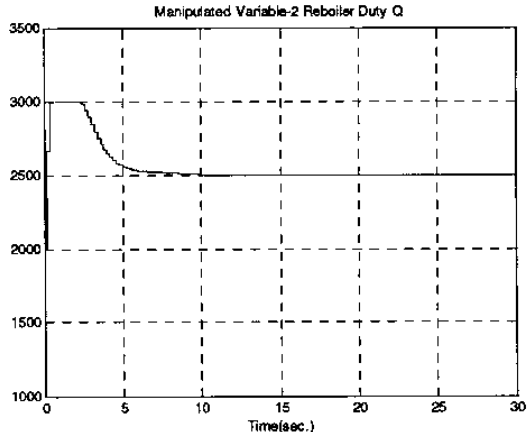

Figure 10 Manipulated Variable Moves for Reboiler Duty $Q$

\section{CONCLUSIONS}

A model predicative controller has been designed and successfully applied on the shell benchmark process. For this purpose the process was identified using standard least squares technique. The overall performance of the controller has been very good and all the variables achieve their desired steady state values. The stringent process constraints have been satisfied and in general the servo behavior of the controller is found to be highly desirable.

\section{REFERENCES}

[1] J. Richalet, A. Rault, J. Papon, "Model Predictive Heuristic Control: Applications to Industrial Processes," Automatica, vol. 14, no. 5, pp. 413-428, 1978.

[2] C.R. Cutler and B. L. Ramaker, "Dynamic Matrix Control - A Computer Control Algorithm," Proceedings JACC, San Francisco, USA, 1980

[3] Enso Ikonen and Kaddour Najim, Advanced Process Identification and Control, Marcel Dekker Inc., New York, 2002.

[4] Barry J. Cott, "Summary of the Process Identification Workshop at the 1992 Canadian Chemical Engineering Conference," Journal of Process Control, vol. 5, no. 3, pp. 109 $113,1995$.

[5] Barry J. Cott, "Introduction of the Process Identification Workshop at the 1992 Canadian Chemical Engineering Conference," Journal of Process Control, vol. 5, no. 3, pp. 67 69, 1995.

[6] Ghassan Murad, Ian Postlethwaite and Da-Wei Gu, "A Discrete Internal Model-Based $H^{\infty}$ Controller and Its Application to a Binary Distillation Column," Journal of Process Control, vol. 7, no. 6, pp. 451-465, 1997.

[7] Mel D. Chan, "Identification of a simulated Shell Distillation Column using Time Series Analysis Methods," Journal of Process Control, vol. 5, no. 3, pp. 99-103, 1995.

[8] Pranob Banerjee, Shrish L. Shah, Shaohua Niu and D. Grant Fisher, "Identification of dynamic models for the Shell Benchmark Problem", Journal of Process Control, vol. 5, no. 2, pp. 85-97, 1995. 